PROCEEDINGS OF THE

AMERICAN MATHEMATICAL SOCIETY

Volume 132, Number 7, Pages 1899-1903

S 0002-9939(04)07316-

Article electronically published on January 23, 2004

\title{
NOTES ON THE LARGEST IRREDUCIBLE CHARACTER DEGREE OF A FINITE GROUP
}

\author{
QIAN GUOHUA
}

(Communicated by Stephen D. Smith)

\begin{abstract}
Let $G$ be a finite group and $b(G)$ the largest irreducible character degree of $G$. In this note, we show the following results: if $b(G)<p^{2}$, then $\left|G: O_{p}(G)\right|_{p} \leq p$; if $b(G)<p^{m}$ and, in addition, $G$ is $p$-solvable with abelian Sylow $p$-subgroup, then $\left|G: O_{p}(G)\right|_{p}<p^{m}$.
\end{abstract}

\section{INTRODUCTION}

Let $b(G)$ be the largest irreducible character degree of the finite group $G$. If $b(G)<p^{2}$ for a prime $p$, then [1, Theorem A] states that $\left|G: O_{p}(G)\right|_{p}<p^{2}$ in case $G$ is $p$-solvable. Our first result shows that the condition $p$-solvable is not necessary.

Theorem 1. Let $b(G)$ be the largest irreducible character degree of a finite group $G$. If $b(G)<p^{2}$ for a prime $p$, then $\left|G: O_{p}(G)\right|_{p} \leq p$.

Recall that in paper [1], the key step is to show that $b(G) \geq p^{2}$ for a finite $p$-solvable group $G$ in which $\left|G: O_{p}(G)\right|_{p}=p^{2}$. Another generalization of the main theorem in [1] is the following result.

Theorem 2. Let $b(G)$ be the largest irreducible character degree of a finite $p$ solvable group $G$. If $G / O_{p}(G)$ possesses an abelian Sylow p-subgroup of order $p^{m}$, then $p^{m} \leq b(G)$.

\section{Proofs of THEOREMS}

Let $H$ and $G / N$ be a subgroup and a quotient group of the group $G$, respectively. It is easy to see that both $b(H)$ and $b(G / N)$ are less than or equal to $b(G)$. So, we can use induction on subgroups and quotient groups in our argument. Applying the classification theorem of finite simple groups, we now prove Theorem 2 ,

Proof of Theorem [. By induction, we may assume that $O_{p}(G)=1$ and $G=$ $O^{p^{\prime}}(G)$. If $M$ is a normal $p^{\prime}$-subgroup of $G$, we may assume that $O_{p}(G / M)$ is nontrivial because otherwise we conclude by induction that $|G / M|_{p} \leq b(G / M)$ and so that $|G|_{p} \leq b(G)$; we are done. It also implies that $\Phi(G)$, the Frattini subgroup of $G$ is trivial.

Received by the editors January 28, 2003 and, in revised form, April 3, 2003.

2000 Mathematics Subject Classification. Primary 20 C15.

Key words and phrases. Finite group, character degree.

Project supported by the National Nature Science Foundation of China and the Nature Science Foundation of Jiangsu Provincial Education Department (03KJB11002). 
Case 1. Suppose that all minimal normal subgroups of $G$ are abelian.

Let $F$ be the Fitting subgroup of $G$. Then $F$ is a nontrivial $p^{\prime}$-subgroup; also, we see that $F$ is abelian because $\Phi(G)=1$. We claim that $C_{G}(F)=F$. If this is not true, we may choose a chief factor $A / F$ of $G$ such that $A \leq C_{G}(F)$. Note that if $A$ is solvable, then the Fitting subgroup of $A$ is equal to $F$ and hence $C_{A}(F) \leq F$, a contradiction. It follows that $A / F$ is nonsolvable. Since $\Phi(A) \leq \Phi(G)$ is trivial, $A$ is split over $F$. Hence we may write $A=Y F$, where $Y \cap F=1$ and $A / F \cong Y$. Since $Y$ centralizes $F$, we conclude that $A=Y \times F$. It is easy to see that $Y \triangleleft G$, and so is a nonsolvable minimal normal subgroup of $G$, which contradicts our assumption.

Let $P$ be a Sylow $p$-subgroup of $G$. Since $C_{G}(F)=F$, we see that the abelian group $P$ acts faithfully on the abelian $p^{\prime}$-group $F$. It is well known that there is $\chi \in \operatorname{Irr}(P F)$ such that $\chi(1)=|P|$. So $b(G) \geq b(P F) \geq \chi(1)=|P|$, and we are done.

Case 2. Suppose that there is a minimal normal subgroup, say $M$, that is nonsolvable.

Clearly, $M$ is a $p^{\prime}$-group and $p$ is an odd prime. Let $A=C_{G}(M)$. Set $|A|_{p}=p^{t}$, $|G / A|_{p}=p^{s}$ where $p^{t}+p^{s}=|G|_{p}$. It is clear that $O_{p}(A)=1$. So by induction, there is $\psi \in \operatorname{Irr}(A)$ such that $\psi(1) \geq p^{t}$.

Let $P$ be a Sylow $p$-subgroup of $G$. Note that $O_{p}(P A M)$ centralizes $M$. It follows that $O_{p}(P A M)=O_{p}(A)$ is trivial. Now if $P A M<G$, then induction yields $b(P A M) \geq|P|$ and so $b(G) \geq|P|$, and we are done. So we may assume that $G=P A M$. Let $M=M_{1} \times M_{2} \times \cdots \times M_{k}$ be the direct product of isomorphic simple groups $M_{i}$. Note that both $N_{G}\left(M_{1}\right)$ and $C_{G}\left(M_{1}\right) \times M_{1}$ contain $A \times M$. Since $P$ is abelian, it forces both $N_{G}\left(M_{1}\right)$ and $C_{G}\left(M_{1}\right) \times M_{1}$ to be normal subgroups of $G$. It is well known that $G / N_{G}\left(M_{1}\right)$ acts faithfully and transitively on $\left\{M_{1}, \ldots, M_{k}\right\}$. Now since $G$ transitively acts on $\left\{M_{1}, \ldots, M_{k}\right\}$, we see that $k=\left|G / N_{G}\left(M_{1}\right)\right|, N_{G}\left(M_{1}\right)=$ $\cdots=N_{G}\left(M_{k}\right), C_{G}\left(M_{1}\right) \times M_{1}=\cdots=C_{G}\left(M_{k}\right) \times M_{k}$. Hence, $C_{G}\left(M_{i}\right) \times M_{i}=A \times M$ for any $1 \leq i \leq k$.

Suppose that $N_{G}\left(M_{1}\right)=C_{G}\left(M_{1}\right) \times M_{1}=A \times M$. Then $k=|G /(A \times M)|=p^{s}$. Let $\lambda_{i} \in \operatorname{Irr}\left(M_{i}\right)$ with degree $\lambda_{i}(1) \geq 3$, and set $\lambda=\lambda_{1} \times \cdots \times \lambda_{k}$. Then $\lambda$ is extendible to $A \times M$. By [4, Corollary 6.17], there is an irreducible constituent $\eta$ of $\lambda^{A \times M}$ with degree $\eta(1)=\lambda(1) \psi(1)$. Thus

$$
b(G) \geq b(A M) \geq \lambda(1) \psi(1) \geq 3^{p^{s}} p^{t} \geq p^{s} p^{t}=|P|,
$$

and we are done.

Suppose that $N_{G}\left(M_{1}\right)>C_{G}\left(M_{1}\right) \times M_{1}=A \times M$. Note that since $N_{G}\left(M_{1}\right) /$ $\left(C_{G}\left(M_{1}\right) \times M_{1}\right)$ is a $p$-group, we see that $p \| O u t\left(M_{1}\right) \mid$. By a well-known theorem (see p. 303 and 304 of [3]), we know that $M_{1}$ is a simple group of Lie type over a field $G F(q)$, where $q=r^{m}$ is a power of prime $r$. Also we know that all elements of $N_{G}\left(M_{1}\right) /(A \times M)$ are field automorphisms of $M_{1}$, and that $p^{e}:=\left|N_{G}\left(M_{1}\right) /(A \times M)\right|$ divides $m$. Let $\lambda_{i}$ be the Steinberg-character of $M_{i}$ and set $\lambda=\lambda_{1} \times \cdots \times \lambda_{k}$. We have $\lambda_{i}(1)=\left|M_{i}\right|_{r} \geq r^{m} \geq r^{p^{e}}>p^{e}$. Thus $\lambda(1)>p^{e k} \geq k p^{e}=|G /(A \times M)|$. Arguing as in the above paragraph, we conclude that $b(G) \geq b(A M) \geq \psi(1) \lambda(1) \geq$ $|P|$; we are done.

Now we begin to prove Theorem 1

Lemma 3. If $G$ is a nonabelian simple group, then Theorem 1 holds. 
Proof. If $G$ is either a simple group of Lie type or one of the sporadic simple groups, then by a well-known theorem of [5], $G$ has an irreducible character $\chi$ of $p$-defect zero. Hence the hypothesis of the theorem yields that $|G|_{p} \leq p$; we are done. Suppose that $G$ is the alternating group $A_{n}$ for $n \geq 5$. If Theorem 1 is not true, then $|G|_{p} \geq p^{2}, p$ is an odd prime and $n \geq 2 p \geq 6$. Corresponding to the partition $(n-3,1,1,1)$, we have the character $\chi_{(n-3,1,1,1)} \in \operatorname{Irr}\left(S_{n}\right)$ with degree $\chi_{(n-3,1,1,1)}(1)=(n-1)(n-2)(n-3) / 6$. Hence $G$ has an irreducible constituent $\psi$ of $\left(\chi_{(n-3,1,1,1)}\right)_{G}$. We see that

$$
p^{2}>\psi(1) \geq(n-1)(n-2)(n-3) / 12 .
$$

This implies that $(2 p-1)(2 p-2)(2 p-3)<12 p^{2}$, and so $p=3$. Then $3^{2}>$ $(n-1)(n-2)(n-3) / 12$ forces that $n \leq 9$. However, for $6 \leq n \leq 9, A_{n}$ always has an irreducible character $\eta$ with degree $\eta(1) \geq p^{2}=9$, a contradiction. By the classification theorem of finite simple groups, the proof is complete.

Lemma 4. If Theorem 1 is not true, to work for a contradiction, we may assume $G$, satisfying the following conditions:

(1) $O_{p}(G)=1 ; G=O^{p^{\prime}}(G)$.

(2) $|G|_{p}=p^{2}$.

(3) If $N$ is a normal $p^{\prime}$-subgroup of $G$, then $O_{p}(G / N)$ is nontrivial.

Proof. (1) By induction, we may assume that our theorem holds for any proper subgroup and proper quotient group. Hence we may assume that $O_{p}(G)=1$ and $G=O^{p^{\prime}}(G)$.

(2) By (1), we may assume that $O_{p}(G)=1$ and $G=O^{p^{\prime}}(G)$. Let $N$ be a minimal normal subgroup of $G$. By Lemma 3, we may assume that $N<G$. Let $A / N=O_{p}(G / N)$. If $A<G$, then induction yields that $|A|_{p},|G / A|_{p} \leq p$. So if our theorem is not true, then we may assume that $|G|_{p}=p^{2}$. In case $A=G$ let $M$ be a maximal normal subgroup of $G$ such that $N \subseteq M$. Then $|G / M|=p$ and by induction we conclude that $|M|_{p} \leq p$. Thus if our theorem is not true, we may also assume that $|G|_{p}=p^{2}$.

(3) If $O_{p}(G / N)$ is trivial for a normal $p^{\prime}$-subgroup $N$ of $G$, then by induction we have $|G / N|_{p} \leq p$ and hence our theorem holds. So we may assume that $O_{p}(G / N)$ is nontrivial.

Proof of Theorem 1. Suppose that the theorem is not true. To work for a contradiction, we may assume that $G$ satisfies all conditions of Lemma 4 By Theorem 2 and Lemma 3 we may also assume that $G$ is neither simple nor $p$-solvable.

Case 1. Suppose that $G$ possesses a minimal normal $p^{\prime}$-subgroup $N$.

Let $M$ be the maximal normal $p^{\prime}$-subgroup of $G$ such that $N \leq M$. Since $G$ is not $p$-solvable, by (2) and (3) of Lemma 4, both $A / M=O_{p}(G / M)$ and $B / N=O_{p}(G / N)$ are of order $p$. We claim that $C_{A}(M) \leq M, C_{B}(N) \leq N$ and $\left|C_{G}(M)\right|_{p}=p$. Suppose that $C_{A}(M) \nsubseteq M$ (or $C_{B}(N) \nsubseteq \mathbb{N}$ ). Then $O_{p}(A)$ (or $\left.O_{p}(B)\right)$ has order $p$, and so $O_{p}(G)>1$, a contradiction. Thus $C_{A}(M) \subseteq M$ and so $\left|C_{G}(M)\right|_{p} \leq p$. Suppose that $\left|C_{G}(M)\right|_{p}=1$. Let $P$ be a Sylow $p$-subgroup of $G$. We see that $P$ acts faithfully on $M$. Then by Theorem 2, there is $\psi \in \operatorname{Irr}(P M)$ with degree $\psi(1) \geq p^{2}$, clearly a contradiction. Hence, $\left|C_{G}(M)\right|_{p}=p$, and since $C_{G}(M) A \triangleleft G$, it follows that $G=A C_{G}(M)$ because $G=O^{p^{\prime}}(G)$ and $\left|G: A C_{G}(M)\right|$ is a $p^{\prime}$-number. Suppose that $M \nsubseteq C_{G}(M)$. We claim that $A \cap C_{G}(M)$ is trivial. Otherwise, we also have $G=A_{1} C_{G}(M)$ where $A_{1} /\left(A \cap C_{G}(M)\right)=O_{p}(G /(A \cap$ 
$\left.C_{G}(M)\right)$ ) is of order $p$ (note that $A \cap C_{G}(M)=C_{A}(M) \leq M \cap C_{G}(M)<M$ and that $\left.A_{1} \cap C_{G}(M)=A \cap C_{G}(M)\right)$. This implies that $A=A_{1}$, a contradiction. Therefore, $A \cap C_{G}(M)$ is trivial and so $G=A \times C_{G}(M)$. Clearly, $O_{p}(A)$ and $O_{p}\left(C_{G}(M)\right)$ are trivial. So we can choose $\chi_{1} \in \operatorname{Irr}(A)$ and $\chi_{2} \in \operatorname{Irr}\left(C_{G}(M)\right)$ such that $\chi_{1}(1)_{p}=\chi_{2}(1)_{p}=p$. Then for $\chi=\chi_{1} \times \chi_{2} \in \operatorname{Irr}(G)$, we have $\chi(1) \geq p^{2}$, a contradiction. Hence $M \leq C_{G}(M)$. Let $L / M$ be a chief factor of $G$ such that $L \leq C_{G}(M)$. By the maximality of $M$, we see that $p$ divides $|L / M|$. Thus $|G: A L|$ is a $p^{\prime}$-number. It follows that $G=A L$ and so $L=C_{G}(M)$ and $L / M$ is a nonabelian simple group with $|L / M|_{p}=p$. Also we see that $L^{\prime}=L$ because $G=O^{p^{\prime}}(G)$. Thus $M$ is a subgroup of the Schur multiplier, say $S$, of the nonabelian simple group $L / M$.

Clearly $N \leq M$ is solvable, and hence $N$ is an elementary abelian $v$-group of order $v^{t}$ for some prime $v$. Note that since a Sylow $p$-subgroup $P_{1}$ of $B$ acts nontrivially on $N$, it forces that $p \mid\left(v^{t}-1\right)$. Clearly $p>2$ because $L / M$ is nonsolvable with $|L / M|_{p}=p$. So $S$ has an elementary abelian subgroup of order $v^{t}$ such that $p \mid\left(v^{t}-1\right)$ where $p \geq 3$. If $L / M$ is either an alternating group $A_{n}$ or one of the sporadic simple groups, consulting the table on p. 302 and 303 of [3], we see that $S$ is a cyclic group and the largest prime divisor of its order is at most 3 , which is impossible. If $G$ is a simple group of Lie type over a field $G F(q)$ where $q=r^{m}$ is a power of prime $r$, then $S=S_{1} \times S_{2}$, where $S_{1}, S_{2}$ are the diagonal multiplier and the exceptional multiplier of $L / M$, respectively. Note that since $S_{1}$ is an $r^{\prime}$-group and $S_{2}$ is an $r$-group, it follows that either $N \leq S_{1}$ or $N \leq S_{2}$.

Suppose that $N \leq S_{2}$. Note that $|N|-1$ has an odd prime divisor $p$. Consulting the table of multipliers of simple groups of Lie type (see [3, p. 302]), we conclude that $L / M$ is one of following groups: $A_{2}(4)$ with $S_{2}=Z_{4} \times Z_{4} ; D_{4}(2)$ with $S_{2}=Z_{2} \times Z_{2} ;{ }^{2} A_{5}(2)$ with $S_{2}=Z_{2} \times Z_{2} ;{ }^{2} B_{2}(8)$ with $S_{2}=Z_{2} \times Z_{2} ;{ }^{2} E_{6}(2)$ with $S_{2}=Z_{2} \times Z_{2}$. In each of these cases, we see that $N$ is an elementary abelian 2-group of order 4 and $p=3$. Checking the degree of the Steinberg-character $\chi$ of $L / M$ in each of these cases, we see that $\chi(1) \geq 3^{2}$, a contradiction.

Suppose that $N \leq S_{1}$. Note that $|N|-1$ has an odd prime divisor $p$. Consulting the table on p. 302 of [3], we see that $L / M$ is either a simple group of type $A_{l}(q)$ with $S_{1}=Z_{(l+1, q-1)}$ or a simple group of type ${ }^{2} A_{l}(q), l \geq 2$ with $S=Z_{(l+1, q+1)}$. In each of these cases, we see that $p \leq|N|-1 \leq l$ and that $L / M$ has the Steinbergcharacter $\chi$ with degree $q^{l(l+1) / 2} \geq q^{p} \geq p^{2}$, a contradiction.

Case 2. Suppose that $G$ has no minimal normal $p^{\prime}$-subgroup.

Let $N$ be a minimal normal subgroup of $G$. By Lemma 3, $N<G$. Since $G=O^{p^{\prime}}(G)$ and $|G|_{p}=p^{2}$, we see that $|N|_{p}=p$ and so that $N$ is a nonabelian simple group.

Suppose that $M=C_{G}(N)>1$. Since $O_{p^{\prime}}(G)=1$ and $O_{p}(G)=1$, we see that $|M|_{p}=p$ and $M$ is also a nonabelian simple group. Then $G=O^{p^{\prime}}(G)$ implies that $G=M \times N$. By [4, Theorem 12.29], we may choose $\chi_{1} \in \operatorname{Irr}(M), \chi_{2} \in \operatorname{Irr}(N)$ such that $\chi_{1}(1), \chi_{2}(1) \geq p$. So $G$ has an irreducible character $\chi=\chi_{1} \times \chi_{2}$ with degree $\chi(1) \geq p^{2}$, a contradiction.

Suppose that $C_{G}(N)=1$. Let $P$ be a Sylow $p$-subgroup of $G$. Let us investigate the subgroup $H=P N$. Clearly, $O_{p}(H)=1$ since $C_{G}(N)=1$. Hence if $H<G$, then induction yields $p^{2}=|H|_{p} \leq b(H) \leq b(G)$, a contradiction. So we may assume $G=P N$. Clearly, $|G / N|=p$ is an odd prime. Let $x \in G / N$ be an outer automorphism of $N$ with order $p$. Applying [3, Theorem 4.239, Theorem 4.240] and the classification theorem of finite simple groups, we conclude that $N$ is a simple 
group of Lie type over a field $G F(q)$, where $q=r^{m}$ is a power of prime $r$. Also, we know that $x$ is either a diagonal automorphism, or a field automorphism, or a graph automorphism.

Suppose that $x$ is a field automorphism. It is well known that $p$, the order of $x$, must divide $m$. Let $\chi$ be the Steinberg-character of $N$. We see that $\chi(1) \geq q=$ $r^{m} \geq 2^{p} \geq p^{2}$, a contradiction.

Suppose that $x$ is a graph automorphism. Consulting the table of automorphisms of the Chevalley groups in [2, p. xvi], and since $p>2$, we see that $N$ is the type $D_{4}(q)$ with $p=3$. In this case, we see that the Steinberg-character $\chi$ of the simple group $N$ has degree $\chi(1) \geq q^{12}>3^{2}$, a contradiction.

Suppose that $x$ is a diagonal automorphism. Consulting the table in [2, p. xvi], we see that $N$ is one of the following types: $A_{l}(q), l \geq 2$ with $p \mid(l+1, q-1)$; ${ }^{2} A_{l}(q), l \geq 2$ with $p \mid(l+1, q+1) ; E_{6}(q)$ with $p \mid(3, q-1),{ }^{2} E_{6}(q)$ with $p \mid(3, q+1)$. Arguing as in the last paragraph of case 1, we conclude that in each of the above cases the Steinberg-character $\chi$ of $N$ has degree $\chi(1) \geq p^{2}$, a contradiction.

\section{REFERENCES}

[1] D. Benjamin, A bound for $\left|G: O_{p}(G)\right|$ in terms of the largest irreducible character degree of a finite p-solvable group $G$, Proc. Amer. Math. Soc. 127:2 (1999), 371-376. MR 99c:20005

[2] J. H. Conway, R. T. Curtis, S. P. Norton, R. A. Parker, and R. A. Wilson, Atlas of finite groups, Oxford Univ. Press (Clarendon), Oxford and New York, 1985. MR 88g:20025

[3] D. Gorenstein, Finite simple groups: An introduction to their classification, Plenum Press, New York, 1982. MR 84j:20002

[4] I. M. Isaacs, Character theory of finite groups, Academic Press, New York, 1976. MR 57:417

[5] W. Willems, Block of defect zero in finite groups of Lie type, J. Algebra 113 (1988), 511-522. MR 89c:20025

Department of Mathematics, Changshu College, Changshu, Jiansu, 215500, People's Republic of China

E-mail address: ghqian2000@yahoo.com.cn 